\title{
Unveiling the Dynamical and Structural Features That Determine the Orientation of the Acceptor Substrate in the Landomycin Glycosyltransferase LanGT2 and Its Variant with C-Glycosylation Activity
}

Fernanda Mendoza ${ }^{* \dagger}$ and Gonzalo A. Jaña ${ }^{* \dagger}$

†Departamento de Ciencias Químicas, Facultad de Ciencias Exactas, Universidad Andres Bello, Autopista Concepción-Talcahuano 7100, Talcahuano, Chile.

\section{Corresponding Authors}

*E-mail: m.mariafernandamendo@uandresbello.edu.

*E-mail: gonzalo.jana@unab.cl. 


\section{TABLE OF CONTENTS}

CGenFF v3.0.1 Topologies and Parameters for TET ........................................................... 4

Binding Affinities of TET in O- and C-LanGT2 ................................................................. 6

Protonation States for Model 1 and 2 in O-LanGT2, and Model 3 and 4 in C-LanGT2 ........... 7

RMSD data for Model 1 and 2 in O-LanGT2, and Model 3 and 4 in C-LanGT2 ..................... 8

Average van der Waals (VdW) Energy Contributions for Loop L51-A62 (O-LanGT2), and

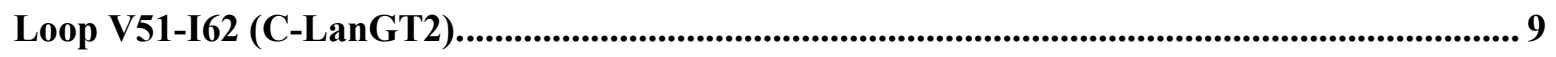




\section{Charges, Atom Types and Topology of Olivose (AOLI)}

Table S1. Atom names, atom types and charges of the residue defined as AOLI, which corresponds to the monosaccharide $\alpha$-olivose.

\begin{tabular}{|c|c|c|}
\hline Atom Name & Atom Type & Charge (a. u.) \\
\hline $\mathrm{C} 1$ & CC3162 & 0.340 \\
\hline H1 & HCA1 & 0.090 \\
\hline O1 & OC311 & -0.650 \\
\hline $\mathrm{HO} 1$ & HCP1 & 0.420 \\
\hline $\mathrm{C} 5$ & CC 3163 & 0.110 \\
\hline H5 & HCA1 & 0.090 \\
\hline O5 & OC3C61 & -0.400 \\
\hline $\mathrm{C} 2$ & CC3161 & -0.180 \\
\hline $\mathrm{H} 21$ & $\mathrm{HCA} 2$ & 0.090 \\
\hline $\mathrm{H} 22$ & 1HCA2 & 0.090 \\
\hline $\mathrm{C} 3$ & CC3161 & 0.140 \\
\hline H3 & HCA1 & 0.090 \\
\hline $\mathrm{O} 3$ & OC311 & -0.650 \\
\hline $\mathrm{HO} 3$ & HCP1 & 0.420 \\
\hline $\mathrm{C} 4$ & CC3161 & 0.140 \\
\hline H4 & HCA1 & 0.090 \\
\hline $\mathrm{O} 4$ & OC311 & -0.650 \\
\hline $\mathrm{HO} 4$ & $\mathrm{HCP} 1$ & 0.420 \\
\hline C6 & CC321 & -0.270 \\
\hline H61 & $\mathrm{HCA} 3$ & 0.090 \\
\hline H62 & HCA3 & 0.090 \\
\hline H63 & HCA3 & 0.090 \\
\hline
\end{tabular}




\section{CGenFF v3.0.1 Topologies and Parameters for TET}

Table S2. Atom names, atom types and charges of the residue defined as TET, which corresponds to the acceptor substrate tetrangulol.

\begin{tabular}{ccc||ccc}
\hline Atom Name & Atom Type & Charge (a. u.) & Atom Name & Atom Type & Charge (a. u.) \\
\hline C1 & CG2R61 & 0.009 & H1 & HGP1 & 0.420 \\
C2 & CG2R61 & -0.115 & O4 & OG311 & -0.532 \\
C3 & CG2R61 & -0.117 & H2 & HGP1 & 0.420 \\
C4 & CG2R61 & 0.023 & H3 & HGR61 & 0.115 \\
C5 & CG2R61 & -0.005 & H4 & HGR61 & 0.115 \\
C6 & CG2R61 & 0.027 & H5 & HGR61 & 0.115 \\
C7 & CG2O5 & 0.408 & H6 & HGR61 & 0.115 \\
C8 & CG2R61 & 0.024 & H7 & HGR61 & 0.115 \\
C9 & CG2O5 & 0.411 & H8 & HGR61 & 0.115 \\
C10 & HCA2 & 0.027 & H9 & HGR61 & 0.115 \\
C11 & CC3161 & 0.110 & H10 & HGA3 & 0.090 \\
C12 & CG2R61 & -0.116 & H11 & HGA3 & 0.090 \\
C13 & CG2R61 & -0.111 & H12 & HGA3 & 0.090 \\
C14 & CG2R61 & -0.116 & & & \\
C15 & CG2R61 & -0.117 & & & \\
C16 & CG2R61 & 0.110 & & & \\
O1 & OG2D3 & -0.459 & & & \\
O2 & OG2D3 & -0.459 & -0.119 & & \\
C17 & CG2R61 & 0.001 & & & \\
C18 & CG2R61 & -0.269 & & \\
C19 & CG331 & -0.530 & & & \\
O3 & OG311 & & & \\
\hline
\end{tabular}


Table S3. New parameters for TET, generated by the CHARMM General Force Field (CGenFF) program version 1.0.0.

\begin{tabular}{|c|c|c|c|c|c|c|}
\hline \multicolumn{5}{|c|}{ Angles } & \multirow{2}{*}{$\frac{\mathbf{k}_{\boldsymbol{\theta}}}{40.00}$} & \multirow{2}{*}{$\frac{\boldsymbol{\theta}_{\mathbf{o}}}{117.20}$} \\
\hline CG2R61 & \multicolumn{2}{|c|}{ CG2O5 } & \multicolumn{2}{|c|}{ CG2R61 } & & \\
\hline \multicolumn{3}{|c|}{ Dihedrals } & & $\mathbf{k}_{\xi}$ & $\mathbf{n}$ & $\delta$ \\
\hline C2GR61 & CG2O5 & C2GR61 & C2GR61 & 1.585 & 2 & 180.00 \\
\hline CG2O5 & CG2R61 & C2GR61 & CG2O5 & 3.100 & 2 & 180.00 \\
\hline CG2O5 & C2GR61 & C2GR61 & OG311 & 2.400 & 2 & 180.00 \\
\hline \multicolumn{5}{|c|}{ Impropers } & $\mathbf{k}_{\psi}$ & $\psi_{0}$ \\
\hline CG2O5 & CG2R61 & CG2R61 & OG2 & & 72.000 & 0.00 \\
\hline
\end{tabular}

The angle (1), dihedral (2) and improper (3) terms have the following expression within the CHARMM potential function:

$$
\begin{gathered}
V_{\theta}=k_{\theta}\left(\theta-\theta_{o}\right)^{2} \\
V_{\text {dihedral }}=k_{\xi}(1+\cos (n \xi-\delta)) \\
V_{\text {improper }}=k_{\psi}\left(\psi-\psi_{o}\right)^{2}
\end{gathered}
$$




\section{Binding Affinities of TET in O- and C-LanGT2}

Table S4. Binding affinity energies $\left(\mathrm{kcal} \cdot \mathrm{mol}^{-1}\right)$ of TET in O-LanGT2 (PDB ID: 4RIF). The selected solution is highlighted in bold.

\begin{tabular}{cc}
\hline Mode & Binding Affinity \\
\hline 1 & -7.4 \\
2 & -7.3 \\
3 & -7.2 \\
4 & -6.2 \\
5 & -6.1 \\
6 & -5.9 \\
7 & $\mathbf{- 5 . 3}$ \\
8 & -5.2 \\
9 & -5.1 \\
10 & -4.7 \\
11 & -4.6 \\
\hline
\end{tabular}

Table S5. Binding affinity energies $\left(\mathrm{kcal} \cdot \mathrm{mol}^{-1}\right)$ of TET in C-LanGT2, PDB code 4RIH. The selected solution is highlighted in bold.

\begin{tabular}{cc}
\hline Mode & Binding Affinity \\
\hline $\mathbf{1}$ & $\mathbf{- 6 . 6}$ \\
2 & -5.8 \\
3 & -5.6 \\
4 & -5.0 \\
5 & -4.1 \\
\hline
\end{tabular}




\section{Protonation States for Model 1 and 2 in O-LanGT2, and Model 3 and 4 in C-LanGT2}

The protonation states of titrable residues were determined by the PROPKA procedure, as it is implemented in the PDB2PQR30 server. Given the fact that the catalytic activity was optimal at $\mathrm{pH}=6.8$ in both the $\mathrm{O}$-and the $\mathrm{C}$-LanGT2 enzymes, the protonation states were calculated at $\mathrm{pH}=7.0$.

Within the active site the only residue with a not standard protonation state is the His283, which is manually defined as HSP. This modeling is adopted based on experimental analyses that indicate $\mathrm{H} 283$ is conserved throughout the GT1 family and has been proposed to stabilize the accumulation of charge on the phosphate during the catalysis (see reference 24). It should be noted that His 283 is rather far from the docking site center $(\sim 10 \AA)$ and it is part of the binding pocket of the donor substrate, in which His283 interacts with the phosphate groups. 


\section{RMSD data for Model 1 and 2 in O-LanGT2, and Model 3 and 4 in C-LanGT2}

The Root Mean Square Deviation (RMSD) of the models 1, 2, 3 and 4 are shown below. Alignment is done using the backbone atoms $(\mathrm{C} \alpha, \mathrm{C}, \mathrm{O}$ and $\mathrm{N})$ of protein residues in each of the models. Thus, the alignment was performed through the MD run trajectory using the first structure of the trajectory as the reference one. The alignment was done according to the VMD guidelines for computing the alignment.
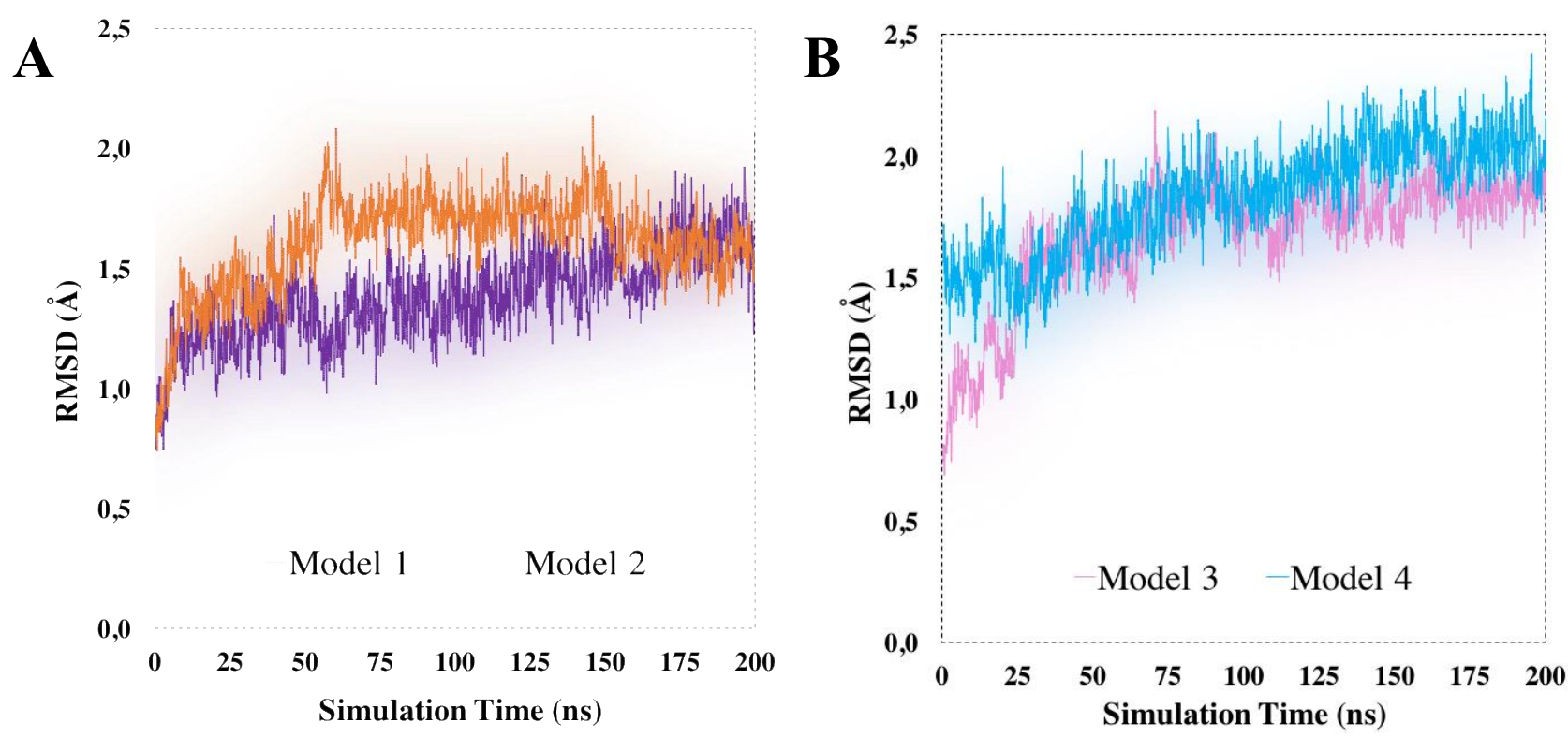

Figure S1. RMSD values calculated during Molecular Dynamics simulations of (A): OLanGT2, and (B): C-LanGT2. 
Average van der Waals (VdW) Energy Contributions for Loop L51-A62 (O-LanGT2), and Loop V51-I62 (C-LanGT2).
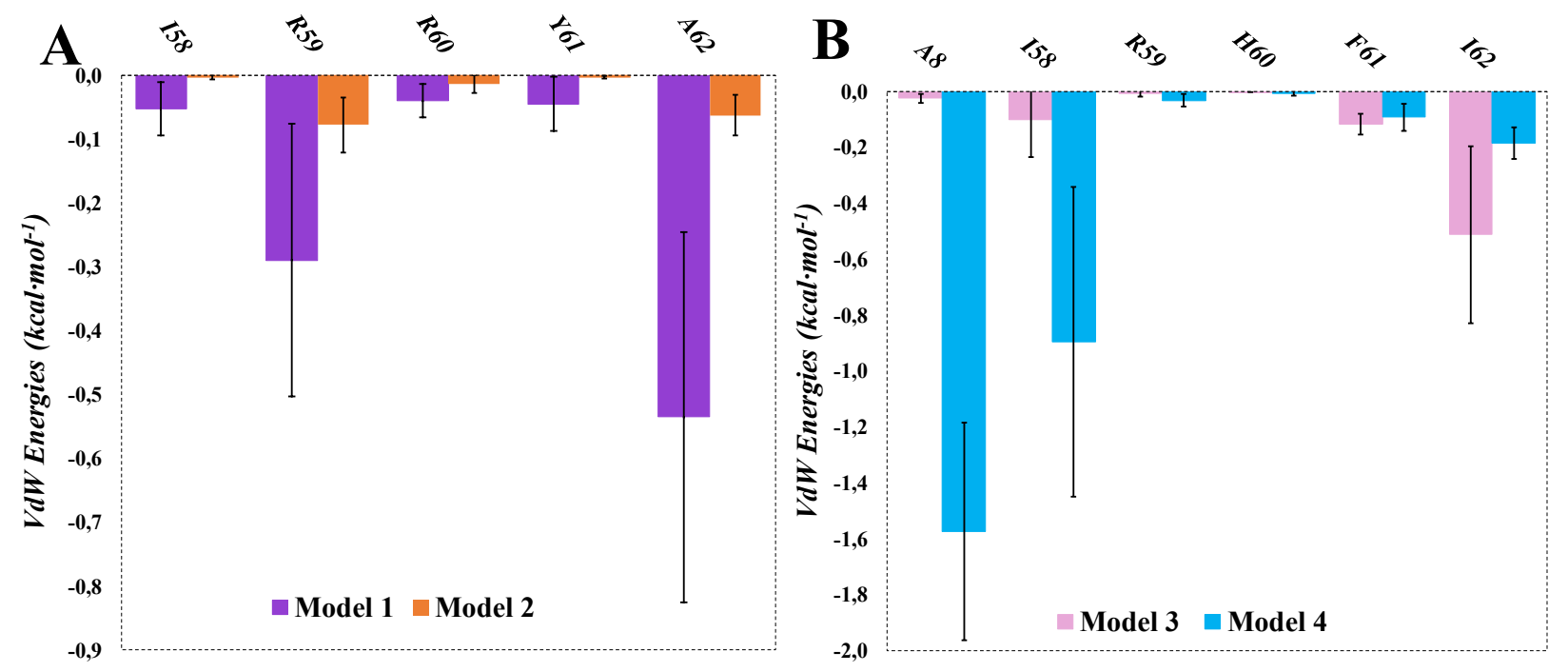

Figure S2. Average VdW energies (columns) and standard deviation (bars) for residues that contribute with a term $\geq 1.5 \times 10^{-3} \mathrm{kcal} \cdot \mathrm{mol}^{-1}$. (A) Model 1 and 2 in O-LanGT2, (B) Model 3 and 4 in C-LanGT2. 